TITLE:

\title{
Some Algebraic Algorithms Based on Head Term Elimination over Polynomial Rings
}

$\operatorname{AUTHOR}(\mathrm{S})$ :

Sasaki, Tateaki

CITATION:

Sasaki, Tateaki. Some Algebraic Algorithms Based on Head Term Elimination over Polynomial Rings. 数理解析研究所講究録 1987, 612: 38-51

ISSUE DATE:

1987-02

URL:

http://hdl.handle.net/2433/99781

RIGHT: 


\title{
Some Algebraic Algorithms Based on
}

Head Term Elimination over Polynomial Rings

\author{
佐火术建昭 \\ Tateaki Sasaki
}

The Institute of Physical and Chemical Research

Wako-shi, Saitama 351-01, Japan

\begin{abstract}
Let $\mathrm{F}_{1}, \ldots, \mathrm{F}_{\mathrm{r}}$ be polynomials in $\mathrm{X}_{1}, \ldots, \mathrm{X}_{\mathrm{n}}$ with coefficients in $\mathrm{K}\left[\mathrm{u}_{1}, \ldots, \mathrm{u}_{\mathrm{m}}\right]$. Many algebraic problems can be reduced to calculating polynomials in $K\left[u_{1}, \ldots, u_{m}\right]$ by eliminating $X_{1}, \ldots, X_{n}$ from $F_{1}, \ldots, F_{r}$. We formulate this elimination in terms of the Gröbner basis of polynomial ideal $\left(\mathrm{F}_{1}, \ldots, \mathrm{F}_{\mathrm{r}}\right)$ over $\mathrm{K}\left[\mathrm{u}_{1}, \ldots, \mathrm{u}_{\mathrm{m}}\right]$. The elimination theory developed is applied to several typical problems. Furthermore, some ideas for making the elimination efficient are presented, with timing data by actual implementation.
\end{abstract}

\section{$\$ 1$. Introduction}

Many algebraic calculations are reduced to the elimination of variables. The conventional elimination method is the leading term elimination. For polynomials $F$ and $G$ in main variable $X$, the leading term elimination is defined by the formula

$$
\frac{1 \mathrm{~cm}}{\ell t(F)} \cdot F-\frac{1 \mathrm{~cm}}{\ell t(G)} \cdot G, \quad \quad \mathrm{lcm}=\operatorname{Lcm}(\ell t(F), \ell t(G)) \text {, }
$$

where $\ell t$ and $\mathrm{LCm}$ denote the "leading term" and "least common multiple", respectively. (The resultant calculation is nothing but a successive application of the leading term elimination.)

Another kind of elimination is the head term elimination which plays an essential role in the construction of Gröbner basis of the polynomial ideal [Buch65]. (For precise definition of "head term", see \$2.) For polynomials F and G with 
coefficients in a field, the head term elimination is defined by the formula

$$
\frac{1 \mathrm{~cm}}{h t(F)} \cdot F-\frac{1 \mathrm{~cm}}{h t(G)} \cdot G, \quad \mathrm{~cm}=\operatorname{Lcm}(h t(F), h t(G)) \text {, }
$$

where ht denotes the head term. (This formula is nothing but the S-polynomial of $\mathrm{F}$ and G.) Note the similarity between the formulas (1) and (2).

Although the leading term elimination is employed in many algorithms, the superiority of head term elimination is being recognized in many calculations. This is because that the head term elimination is more general than the leading term elimination in that the latter can be attained by successive application of the former. Furthermore, we can determine the term ordering variously for head term elimination, while the term ordering for leading term elimination is unique when we have determined the main variable.

In many algorithms, the following elimination is required: given polynomials $F_{1}$, $\ldots, F_{r}$ in $R\left[X_{1}, \ldots, X_{n}\right]$, where $R=K\left[u_{1}, \ldots, u_{m}\right]$, calculate polynomial(s) in $u_{1}$, $\ldots u_{m}$ by eliminating $X_{1}, \ldots, X_{n}$ from $F_{1}, \ldots, F_{r}$. Following Buchberger [Buch65,84], we formulate this elimination as a construction of Gröbner basis of the polynomial ideal over ring $\mathrm{R}=\mathrm{K}\left[\mathrm{u}_{1}, \ldots, \mathrm{u}_{\mathrm{m}}\right]$.

In \$2, we develop a theory of Gröbner basis of the polynomial ideal over polynomial ring $R$. Some typical problems which can be solved simply and efficiently by the use of head term elimination in $R\left[X_{1}, \ldots, X_{n}\right]$ are described in $\$ 3$. In many cases of elimination, we are unnecessary to calculate the full set of Gröbner basis but to calculate only a subset of Gröbner basis. Exploiting this, we can avoid unnecessary computation and save the time largely, which is explained in $\$ 4$. The algorithms to be presented in this paper have been implemented on the Japanese algebra system GAL, and $\$ 4$ shows the timing data also.

\section{\$2. Gröbner basis of polynomial ideal over polynomial ring}

After Buchberger's pioneering work on Gröbner basis [Buch65], which is for polynomials with coefficients in a field, various extensions have been made. As for extending the coefficient domain, Lauer [Lauer76] and Buchberger [see Buch84] 
developed Gröbner basis theories over the integer ring, and Kandri-Rody and Kapur extended them to Euclidean rings [Ka\&Ka84]. In this section, we extend the coefficient domain to polynomial rings, which is straightforward.

We denote the set of nonnegative integers by $\mathbb{Z}_{0}$ and the Cartesian product $\mathbb{Z}_{0} \times \cdots \times \mathbb{Z}_{0}$ by $\mathbb{Z}_{0}^{\mathrm{r}}$. Let $\mathrm{K}$ be a field and $\mathrm{R}$ a ring $\mathrm{K}\left[\mathrm{u}_{1}, \ldots, \mathrm{u}_{\mathrm{m}}\right]$. We abbreviate the rings $K\left[u_{1}, \ldots, u_{m}\right]$ and $R\left[X_{1}, \ldots, X_{n}\right]$ to $K[u]$ and $R[X]$, respectively. Similarly, we abbreviate monomials $c_{1}^{a_{1}} \cdots u_{m}^{a_{m}}$ and $c X_{1}^{A_{1}} \cdots X_{n}^{A_{n}}$, where $c \varepsilon K$ and $c \in R$, to $\mathrm{cu}^{\mathrm{a}}$ and $\mathrm{CX}^{\mathrm{A}}$, respectively. The ideal generated by $\mathrm{F}_{1}, \ldots, \mathrm{F}_{\mathrm{r}}$ is denoted by $\left(\mathrm{F}_{1}, \ldots, \mathrm{F}_{\mathrm{r}}\right)$. Furthermore, we denote the pair of $\mathrm{a}$ and $\mathrm{b}$ by $\langle\mathrm{a}, \mathrm{b}\rangle$.

$\underline{\text { Definition } 1}$ [order $>$ for elements of $\mathbb{Z}_{0}^{\mathrm{r}}$ ]. Let $\mathrm{a}=\left(\mathrm{a}_{1}, \ldots, \mathrm{a}_{\mathrm{r}}\right)$ and $\mathrm{b}=$ $\left(\mathrm{b}_{1}, \ldots, \mathrm{b}_{\mathrm{r}}\right)$ be elements of $\mathbb{Z}_{0}^{\mathrm{r}}$. We define $\mathrm{a}>\mathrm{b}$ iff there exists an integer $\mathrm{k}$ such that $a_{k}>b_{k}$ and $a_{i}=b_{i}, i=1, \ldots, k-1$ when $k>1$. //

Definition 2 [total degree]. Let $t=c u_{1}^{a_{1}} \cdots u_{m}^{a_{m}}$ and $T=c X_{1}^{A_{1}} \cdots X_{n}^{A_{n}}$, with $c \varepsilon \mathrm{K}$ and $\mathrm{C} \varepsilon \mathrm{R}$, be monomials in $\mathrm{K}[\mathrm{u}]$ and $\mathrm{R}[\mathrm{X}]$, respectively. Total degrees of $\mathrm{t}$ and $T$, which are abbreviated to tdeg(t) and $\operatorname{Tdeg}(T)$, respectively, are $a_{1}+\cdots+$ $a_{m}$ and $A_{1}+\cdots+A_{n} \cdot / /$

Definition 3 [order $>$ for monomials in $K[u]$ and $R[X]$ ]. Let $t_{a}=c_{a} u_{1}^{a_{1}} \cdots u_{m}^{a_{m}}$ and $t_{b}=c_{b} u_{1}^{b} \cdots u_{m}^{b_{m}}$, with $c_{a}, c_{b} \in K$, be monomials in $K[u]$. The lexicographic order $>$ between $t_{a}$ and $t_{b}$ is defined as $t_{a} \gg t_{b}$ iff $\left(a_{1}, \ldots, a_{m}\right)>\left(b_{1}, \ldots, b_{m}\right)$. The total-degree order $>$ is defined as $t_{a} \triangleright t_{b}$ iff $\left(\operatorname{tdeg}\left(t_{a}\right), a_{1}, \ldots, a_{m}\right)>$ $\left(\operatorname{tdeg}\left(t_{b}\right), b_{1}, \ldots, b_{m}\right)$ Let $T_{A}=C_{A} X_{1}^{A_{1}} \cdots X_{n}^{A_{n}}$ and $T_{B}=C_{B} X_{1}^{B_{1}} \cdots X_{n}^{B_{n}}$, with $C_{A}, C_{B} \in R$, be monomials in $R[X]$. We define the lexicographic order $D$ between $T_{A}$ and $T_{B}$ by $n$-tupples $\left(A_{1}, \ldots, A_{n}\right)$ and $\left(B_{1}, \ldots, B_{n}\right)$ and the total-degree order $B$ by $(n+1)$-tupples $\left(\operatorname{Tdeg}\left(T_{A}\right), A_{1}, \ldots, A_{n}\right)$ and $\left(\operatorname{Tdeg}\left(T_{B}\right), B_{1}, \ldots, B_{n}\right)$, respectively, just the same as for the monomials in $\mathrm{K}[\mathrm{u}]$.

Definition 4 [head term]. Let $\mathrm{f}$ be a polynomial in $\mathrm{K}[\mathrm{u}]$ and $\mathrm{t}$ the highest order monomial in $f$. We call $t$ the head term of $f$ and abbreviate to ht(f). Similarly, the head term is defined for polynomial $F$ in $R[X]$, with abbreviation $H t(F)$.

Definition 5 [head power product, head coefficient]. Let $f \varepsilon K[u]$ and $h t(f)=c^{a}$, with $c \in K$. We call $u^{a}$ and $c$ the head power product of $f$ and the head coefficient 
of $f$, respectively, and abbreviate to $h p(f)$ and $h c(f)$. Similarly, for polynomial $F$ in $R[X]$ with $H t(F)=C X^{A}, C \varepsilon R$, we call $X^{A}$ and $C$ the head power product of $F$ and head coefficient of $\mathrm{F}$, respectively, with abbreviations $\mathrm{Hp}(\mathrm{F})$ and $\mathrm{Hc}(\mathrm{F})$.

Definition 6 [order $\gg$ for monomials in $K[u, X]$ ]. Let $T_{a}=c_{a} u^{a} X^{A}$ and $T_{b}=c_{b} u^{b} X^{B}$. with $c_{a}, c_{b} \in K$, be monomials in $K[u, X]$. We define $T_{a} \gg T_{b}$ iff either $X^{A} \gg X^{B}$ or $A=B$ and $u^{a} \quad D u^{b}$.

Remark. We can define $\gg$ similarly as $\gg$. For example, if $\triangleright$ is the total-degree order in both $\mathrm{K}[\mathrm{u}]$ and $\mathrm{R}[\mathrm{X}]$, then we can define the order $\gg$ for monomial $c u_{1}^{a_{1}} \cdots u_{m}^{a_{m}} \cdot X_{1}^{A_{1}} \cdots X_{n}^{A_{n}}$, with $c \varepsilon K$, by the order of $(m+n+2)$-tupple $\left(\Sigma A_{j}, A_{1}, \ldots, A_{n}, \sum a_{i}, a_{1}, \ldots, a_{m}\right)$.

Definition 7 [abbreviations Hhp and Hhc]. We abbreviate $\mathrm{hp}(\mathrm{Hc}(\mathrm{F})) \cdot \mathrm{Hp}(\mathrm{F})$ and $\mathrm{hc}(\mathrm{Hc}(\mathrm{F}))$ to $\mathrm{Hhp}(\mathrm{F})$, and $\mathrm{Hhc}(\mathrm{F})$, respectively. //

Definition $8[S$-polynomial in $R[X]=K[u][X]]$. Let $F, G \in R[X]$. The S-polynomial of $F$ and $G$, to be abbreviated to $\operatorname{Spol}(F, G)$, is defined as

$$
\operatorname{Spol}(F, G)=\frac{L C M}{\operatorname{Hhp}(F)} \cdot F-\frac{L C M}{\operatorname{Hhp}(G)} \cdot \frac{\operatorname{Hhc}(F)}{\operatorname{Hhc}(G)} \cdot G
$$

where $\mathrm{LCM}=\operatorname{Lcm}(\operatorname{Hhp}(\mathrm{F}) \cdot \operatorname{Hhp}(\mathrm{G}))$.//

Example 1. Let $\gg$ be the total-degree order and

$$
\begin{aligned}
& F=\left(2 u_{1}^{2} u_{2}+3 u_{2}^{2}+2 u_{1}\right) X_{1} X_{2}^{2}+\text { (terms of less order). } \\
& G=\left(3 u_{1} u_{2}^{2}-2 u_{1}^{2}+3 u_{2}\right) X_{1}^{2} X_{2}+\text { (terms of less order). }
\end{aligned}
$$

Then, $\operatorname{Hhp}(F)=u_{1}^{2} u_{2} \cdot X_{1} X_{2}^{2}$ and $\operatorname{Hhp}(G)=u_{1} u_{2}^{2} \cdot X_{1}^{2} X_{2}$, and

$$
\begin{aligned}
\operatorname{Spol}(F, G) & =u_{2} X_{1} \cdot F-u_{1} X_{2}(2 / 3) \cdot G \\
& =\left((4 / 3) u_{1}^{3}+3 u_{2}^{3}\right) X_{1}^{2} X_{2}^{2}+\text { (terms of less order). }
\end{aligned}
$$

Definition 9 - $[M$-reduction in $R[X]]$. Let $F, G \in R[X]$. If a monomial $T$ of $F$ is such that $\mathrm{T}=\mathrm{X}^{\mathrm{A}} \mathrm{Hp}(\mathrm{G}) \cdot\left\{\cdots+\mathrm{cu}^{\mathrm{a}} \cdot \mathrm{ht}(\mathrm{Hc}(\mathrm{G}))+\cdots\right\}$, then $F^{\prime}=F-\mathrm{cu}^{\mathrm{a}} \mathrm{X}^{\mathrm{A}} \cdot \mathrm{G}$ is a procedure of replacing $T$ by lower order terms. This procedure is called the M-reduction of $F$ by $G$ and expressed as $F \underset{G}{\longrightarrow} F^{\prime}$. //

Remark. The M-reduction by $G$ is nothing but the rewriting of terms by the rule $\operatorname{Hht}(G) \longrightarrow G-\operatorname{Hht}(G)$, where $\operatorname{Hht}(G)=\operatorname{Hhc}(G) \cdot \operatorname{Hhp}(G)$. For instance, if $G$ is given as in Example 1, the replacing rule is 


$$
3 u_{1} u_{2}^{2} \cdot x_{1}^{2} x_{2} \rightarrow\left(2 u_{1}^{2}-3 u_{2}\right) x_{1}^{2} x_{2}-\text { (terms of less order). }
$$

Remark. When $\operatorname{Hhp}(G) \mid \operatorname{Hhp}(F)$, the $\operatorname{Spol}(F, G)$ in Def. 8 is nothing but the M-reduction of (head term of) $F$ by $G$.

Definition 10 [normal form in $R[X]$. Let $\Gamma=\left\{G_{1} \ldots, G_{s}\right\}$ be a subset of $R[X]$. When $F$ is $M$-reduced by $G_{1}, \ldots, G_{s}$ as far as possible to $\tilde{F}$, we call $\tilde{F}$ the normal form of $F$ w.r.t. $\Gamma$ and express as $F \underset{\Gamma}{\longrightarrow} \tilde{F}$. //

Definition 11 [Gröbner basis in $R[X]]$. Let $\left\{F_{1}, \ldots, F_{r}\right\}$ and $\Gamma=\left\{G_{1}, \ldots, G_{s}\right\}$ be subsets of $R[X]$. The set $\Gamma$ is a Gröbner basis of ideal $\left(F_{1}, \ldots, F_{r}\right)$ if the following two conditions are satisfied:

(1) $\left(F_{1}, \ldots, F_{r}\right)=\left(G_{1}, \ldots, G_{s}\right)$,

(2) for any pair $\left\langle G_{i}, G_{j}\right\rangle$ in $\Gamma, \operatorname{Spol}\left(G_{i}, G_{j}\right) \underset{\Gamma}{\longrightarrow} 0$. //

Given a set $\left\{F_{1}, \ldots, F_{r}\right\}$ in $R[X]$, we can construct a Gröbner basis $\left\{G_{1}, \ldots, G_{s}\right\}$ of ideal $\left(\mathrm{F}_{1}, \ldots, \mathrm{F}_{\mathrm{r}}\right)$ by Buchberger's celeborated procedure [Buch65].

Buchberger's procedure.

$$
\begin{aligned}
& \text { Input : Polynomials } \mathrm{F}_{1}, \ldots, \mathrm{F}_{\mathrm{r}} \text { in } \mathrm{R}[\mathrm{X}] \text {; } \\
& \text { Output: Gröbner basis of }\left(F_{1} \ldots . . F_{r}\right) \text { in } R[X] \text {; } \\
& \Gamma:=\left\{G_{1}:=F_{1}, \cdots, G_{r}:=F_{r}\right\} ; \\
& P:=\left\{\left\langle G_{i}, G_{j}\right\rangle \mid G_{i}, G_{j} \in \Gamma, G_{i} \neq G_{j}\right\} ; \\
& \text { while } P \neq \phi \text { do begin } \\
& \left\langle G_{i}, G_{j}\right\rangle:=\text { a pair in } P \text {; } \\
& P:=P-\left\{\left\langle G_{i}, G_{j}\right\rangle\right\} \text {; } \\
& \operatorname{Spol}\left(G_{i}, G_{j}\right) \underset{\Gamma}{\longrightarrow} G \text {; } \\
& \text { if } G \neq 0 \text { then begin } \\
& \begin{array}{l}
P:=P \cup\left\{\left\langle G_{i}, G>\right| G_{i} \varepsilon \Gamma\right\} ; \\
\Gamma:=\Gamma \cup\{G\} ;
\end{array}
\end{aligned}
$$

end; end;

return $\Gamma ; / /$

Theorem. The above procedure terminates and the basis $\left\{G_{1}, \ldots, G_{s}\right\}$ constructed is a Gröbner basis of $\left(F_{1}, \ldots . F_{r}\right)$.

Proof. Let us consider that $F_{1}, \ldots, F_{r}$ and $G_{1}, \ldots, G_{s}$ are elements in $K[u, X]$ 
with the monomial order $\gg$ defined in Def. 6 (see Remark to Def. 6, also). Then, definitions of S-polynomial in Def. 8 and $M$-reduction in Def. 9 are the same as conventional ones in $\mathrm{K}[\mathrm{u}, \mathrm{X}]$. Hence, the proof for the conventional Gröbner basis over the field can be applied to prove the theorem. //

Corollary. Let $\Gamma=\left\{G_{1}, \ldots, G_{s}\right\}$ be a Gröbner basis of $\left(F_{1}, \ldots, F_{r}\right)$, then

(1) for any $F$ in $\left(F_{1}, \ldots, F_{r}\right), F \underset{\Gamma}{\longrightarrow} 0$.

(2) for any $F$ in $R[X]$, normal form of $F$ w.r.t. $\Gamma$ is unique,

(3) $\Gamma \mathrm{K}[\mathrm{u}]$ is a Gröbner basis of ideal $\left(\mathrm{F}_{1}, \ldots, \mathrm{F}_{\mathbf{r}}\right) \cap \mathrm{K}[\mathrm{u}] . / /$

Remark. Let $E$ be a Euclidean polynomial ring with variable $u$. Since $E$ can be regarded as $K[u]$ with $K$ a field, there is a simple relationship between $S$-polynomials in $E[X]$ and $R[X]$. In $E$, we can define the order $P$ by the degree of variable $u$ and perform the reduction by the division operation. Let $f$ and $g$ be elements of $E$ such that $\operatorname{deg}(f) \geqq \operatorname{deg}(g)$, then there exist $q$ and $r$ in $E$ such that

$$
f=q g+r, \operatorname{deg}(r)<\operatorname{deg}(g)
$$

The $\mathrm{q}$ is called the quotient and denoted by quo $(f, g)$. For elements $F$ and $G$ in $E[X]$ such that $\operatorname{deg}(\mathrm{Hc}(\mathrm{F})) \geq \operatorname{deg}(\mathrm{Hc}(\mathrm{G}))$, we define the $\mathrm{S}$-polynomial of $\mathrm{F}$ and $\mathrm{G}$ as

$$
\operatorname{Spol}(F, G)=\frac{L_{C M}^{\prime}}{H p(F)} \cdot F-\frac{L^{\prime} M^{\prime}}{H p(G)} \cdot q u o(H c(F), H c(G)) \cdot G,
$$

where $\mathrm{LCM}^{\prime}=\operatorname{Lcm}(\mathrm{Hp}(\mathrm{F}), \mathrm{Hp}(\mathrm{G}))$.

Let $\delta=\operatorname{deg}(\mathrm{Hc}(F))-\operatorname{deg}(\operatorname{Hc}(G))$. When $F, G \in E[X]$ and $\delta \geqq 0$, we have $h p(H c(F))=$ $\operatorname{Lcm}(\mathrm{hp}(\mathrm{Hc}(\mathrm{F})), \mathrm{hp}(\mathrm{Hc}(\mathrm{G})))$ and

$$
\frac{\mathrm{LCM}}{\operatorname{Hhp}(F)}=\frac{\mathrm{LCM}^{\prime}}{\operatorname{Hp}(F)}, \frac{\mathrm{LCM}}{\operatorname{Hhp}(G)}=\frac{\mathrm{LCM}^{\prime}}{\mathrm{Hp}(G)} \cdot \mathrm{u}^{\delta} .
$$

Hence, by eliminating the head term (= leading term) of $\mathrm{Hc}(\mathrm{F})$ by $\mathrm{Hc}(\mathrm{G})$ successively, we can derive (4) from (3).

\section{§3. Applications of head term elimination}

As we have seen in the Theorem in $\$ 2$, successive application of the head term elimination terminates and we can use Buchberger's procedure as a general elimination procedure. Since the elimination is a very elementary operation, the head 
term elimination will be applied to various algebraic calculations.

We note that, in Buchberger's procedure above, we may choose the term order $\triangleright$ and the pair $\left\langle G_{i}, G_{j}\right\rangle$ arbitrarily. Actually, the efficiency of elimination depends on the choice strongly, and we select the following choices.

Choice 1. We set $\triangleright$ to the total-degree order as far as possible. This is because the total-degree order is much more desirable than the lexicographic order for efficient elimination, as is well-known empirically.

Choice 2. The $G_{i}$ and $G_{j}$ are chosen to be as low order elements as possible.

The importance of this choice will become clear in the next section.

With these choices in mind, we describe four typical applications of the head term elimination in $\mathrm{K}[\mathrm{u}][\mathrm{X}]$, below.

\subsection{Solving a system of algebraic equations}

Consider solving a system of algebraic equations

$$
\left\{F_{1}=0, \cdots, F_{r}=0\right\} \text {, }
$$

where $F_{i} \in K[X], i=1, \ldots, r$, and we assume that the dimension of solution space is 0. (Usually we set $n=r$, but this restriction is not always necessary.) Most algebraic methods for solving (5) are such that an equation in a single variable, $X_{1}$ for example, is derived by eliminating $X_{2}, \ldots, X_{n}$. One practical method performs this elimination by calculating a Gröbner basis of $\left(F_{1}, \ldots, F_{r}\right)$ with the ordering $X_{n} \triangleright \cdots \triangleright X_{1}$ (i.e., the lexicographic order).

Some authors proposed to solve the system (5) by calculating a Gröbner basis with total-degree order. The method is based on the following algorithm.

Algorithm (see [Buch84], Method 6.12).

Input : Gröbner basis $\Gamma=\left\{G_{1}, \ldots, G_{s}\right\}$ with the total-degree order;

Output: Minimum degree polynomial $Q\left(X_{1}\right)$ such that $Q\left(X_{1}\right) \&\left(G_{1}, \ldots, G_{s}\right)$;

Method: Putting $Q=a_{k} X_{1}^{k}+a_{k-1} x_{1}^{k-1}+\cdots+a_{0}$, where $a_{i}, i=0, \ldots, k$, are numeric unknowns and $k$ is an adequate upper bound, construct a system of linear equations on $a_{i}$ by requesting that $Q \underset{\Gamma}{\longrightarrow} 0$. Then, determine $a_{i}$, $\mathrm{i}=0, \ldots, \mathrm{k}$, by solving the system.

By this algorithm, we can calculate an equation $Q_{1}\left(X_{1}\right)=0$ which gives the 
values $\mathrm{X}_{1}$ of the roots of (5). In order to determine the values of other variables, Moritsugu proposed the following method [Mori86]: construct polynomials $Q_{i}\left(X_{1}\right), i=2, \ldots, n$, such that the values of $X_{i}$ are determined by the values of $X_{1}$ as $X_{i}=Q_{i}\left(X_{1}\right)$. The $Q_{i}, i=2, \ldots, n$, can be determined, so long as they exist, by modifying the above algorithm slightly (i.e., requesting that $X_{i}-Q_{i} \longrightarrow \Gamma \quad 0$ ). Moritsugu's method is quite useful, in particular when calculating the roots by solving $Q_{1}\left(X_{1}\right)=0$ numerically because the number of arithmetic operations is quite small in his method compared with other methods. However, it suffers from a defect that polynomials $Q_{i}\left(X_{1}\right)$ do not always exist. In such a case, we must search for quadratic form $X_{i}^{2}+Q_{i 1}\left(X_{1}\right) X_{i}+Q_{i 0}\left(X_{1}\right)=0$, or even higher degree forms.

The above method is simple, but our method below is even simpler (and will be efficient). We calculate polynomials $Q_{1}\left(X_{1}\right)$ and $Q_{i}\left(X_{i}, X_{1}\right), i=2, \ldots, n$, directly, where $Q_{1}$ and $Q_{1}$ satisfy $Q_{1}\left(X_{1}\right)=0$ and $Q_{1}\left(X_{i}, X_{1}\right)=0$, respectively, for the values $X_{1}$ and $X_{i}$ of the roots of (5).

Algorithm A (reducing a system of algebraic equations).

Input : Polynomials $\mathrm{F}_{1}, \ldots, \mathrm{F}_{\mathrm{r}}$ in $\mathrm{K}[\mathrm{X}]$;

Output: Polynomials $Q_{1}\left(X_{1}\right)$ and $Q_{i}\left(X_{i}, X_{1}\right), i=2 \ldots, n$, such that $Q_{1}, Q_{i} \varepsilon$ $\left(F_{1}, \ldots, F_{r}\right)$, with as small $\operatorname{deg}_{\mathbf{X}_{\mathbf{i}}}\left(Q_{\mathbf{i}}\right)$ as possible;

Step 1: Treat $\mathrm{F}_{1}, \ldots, \mathrm{F}_{\mathrm{r}}$ as elements in $\mathrm{R}\left[\mathrm{X}_{2}, \ldots, \mathrm{X}_{\mathrm{n}}\right], \mathrm{R}=\mathrm{K}\left[\mathrm{X}_{1}\right]$, and calculate a Gröbner basis $\Gamma_{1}$ of $\left(F_{1}, \ldots ., F_{r}\right)$ with the total-degree order;

Step 2: For each $i, 2 \leqq i \leqq n$, treat $\mathrm{F}_{1}, \ldots, \mathrm{F}_{\mathrm{r}}$ as elements in $R\left[X_{2}, \ldots, X_{i-1}, X_{i+1}, \ldots, X_{n}\right], R=K\left[X_{1}, X_{i}\right]$, and calculate a Gröbner basis $\Gamma_{i}$ of $\left(F_{1}, \ldots, F_{r}\right)$ with the total-degree order for $X_{2}, \ldots, X_{i-1}, X_{i+1}, \ldots, X_{n}$ and the lexicographic order for $\mathrm{X}_{\mathrm{i}}$ and $\mathrm{X}_{1}$;

Return: Return $Q_{1}$ and $Q_{i}, i=2, \ldots, n$, where $Q_{1} \varepsilon \Gamma_{1} \cap K\left[X_{1}\right], Q_{i} \varepsilon \Gamma_{i} \cap K\left[X_{1}, X_{i}\right]$ and $\operatorname{deg}_{X_{i}}\left(Q_{1}\right)$ is the smallest.

\subsection{Calculating U-resultant}

A classical but theoretically satisfactory method for solving a system of algebraic equations is the U-resultant method. Let the system to be solved be (5). Adding another equation $F_{0}=0$ to (5), where 


$$
\mathrm{F}_{0}=\mathrm{u}_{0}+\mathrm{u}_{1} \mathrm{X}_{1}+\cdots+\mathrm{u}_{\mathrm{n}} \mathrm{X}_{\mathrm{n}} \text {. }
$$

we can calculate the so-called U-resultant. The U-resultant is a homogeneous polynomial in $u_{0}, u_{1}, \ldots, u_{n}$ and it is a product of linear factors:

$$
U\left(u_{0}, u_{1}, \ldots, u_{n}\right)=\prod_{k=1}^{\nu}\left(c_{k 0} u_{0}+c_{k 1} u_{1}+\cdots+c_{k n} u_{n}\right) \text {, }
$$

where $c_{k i} \in C$ (the field of complex numbers). With this factored form of the U-resultant, the set of roots $\left(X_{1}, \ldots, X_{n}\right)$ of $(5)$ is given by

$$
\left\{\left(c_{k 1} / c_{k 0}, \cdots, c_{k n} / c_{k 0}\right) \mid c_{k 0} \neq 0,1 \leqq k \leqq \nu\right\} .
$$

Furthermore, the multiplicity of a root is given by the multiplicity of the same linear factor in $U$. Hence, this method is mathematically complete.

The classical method of calculating the U-resultant is inefficient. In 1977. Lazard presented a practical method [Laza77], and Lazard's method has been made efficient by Kobayashi et al. by using the Gröbner basis [K\&F\&F86]. (This paper also presents a practical method for factoring the U-resultant into linear factors.) However, the calculation is still complicated and time consuming.

Our method using the head term elimination is quite simple, yet it is efficient for small-sized problems. (For large-sized problems, the method of Kobayashi et al. will be better.) Our method calculates the U-resultant by eliminating $X_{1}, \ldots, X_{n}$ from the system of algebraic equations $\left\{F_{0}=0, F_{1}=0, \cdots, F_{r}=0\right\}$.

Algorithm B (U-resultant).

Input $: F_{1}, \ldots, F_{r}$ in $K[X]$ and indeterminates $u_{0}, u_{1}, \ldots, u_{n}$;

Output: U-resultant of $\left\{F_{1}=0, \cdots, F_{r}=0\right\}$;

Method: Treat $F_{0}, F_{1}, \ldots, F_{r}$ as elements in $R[X], R=K[u]$, and calculate a Gröbner basis $\Gamma$ of ideal $\left(F_{0}, F_{1}, \ldots, F_{r}\right)$ with the total-degree order for both $\mathrm{X}_{1}, \ldots, \mathrm{X}_{\mathrm{n}}$ and $\mathrm{u}_{0}, \ldots, \mathrm{u}_{\mathrm{n}}$;

Return: Return the lowest order element $G$ such that $G \varepsilon \Gamma \cap K[u]$. //

\subsection{Calculating algebraic relations}

Let $\mathrm{P}_{1}, \ldots, \mathrm{P}_{\mathrm{r}}$ be polynomials in $\mathrm{K}[\mathrm{X}]$, and let $\rho\left(\mathrm{u}_{1}, \ldots, \mathrm{u}_{\mathrm{r}}\right)$ be a polynomial in $\mathrm{K}\left[\mathrm{u}_{1}, \ldots, \mathrm{u}_{\mathrm{r}}\right]$. If $\rho$ satisfies $\rho\left(\mathrm{P}_{1}, \ldots, \mathrm{P}_{\mathrm{r}}\right)=0$, then $\rho$ is called an algebraic relation of $P_{1}, \ldots, P_{r}$.

Calculation of algebraic relations of given polynomials $P_{1}, \ldots, P_{r}$ are simple 
in principle: we have only to eliminate variables $X_{1}, \ldots, X_{n}$ from the set of polynomials $\left\{\mathrm{P}_{1}-\mathrm{u}_{1}, \cdots, \mathrm{P}_{\mathrm{r}}-\mathrm{u}_{\mathrm{r}}\right\}$. The use of Buchberger's procedure to calculate algebraic relations is described in [Ar\&Se84]. This method calculates a Gröbner basis in $K\left[u_{1}, \ldots, u_{r}, X_{1}, \ldots, X_{n}\right]$. In order to eliminate $X_{1}, \ldots, X_{n}$ first, one usually employs the lexicographic order for $X_{n}, \ldots, X_{1}, u_{r}, \ldots, u_{1}$. If we calculate a Gröbner basis in $R[X]$, where $R=K\left[u_{1}, \ldots, u_{r}\right]$, then we can employ the total-degree order. Note that, with a Gröbner basis calculation, we can calculate a complete set of generators of the algebraic relations (cf. Corollary to Theorem in $\$ 2$ ).

\section{Algorithm C (algebraic relations).}

Input : Polynomials $\mathrm{P}_{1}, \ldots, \mathrm{P}_{\mathrm{r}}$ in $\mathrm{K}[\mathrm{X}]$;

Output: Gröbner basis of the ideal generated by algebraic relations;

Method: Treat $\mathrm{P}_{1}-\mathrm{u}_{1}, \cdots, \mathrm{P}_{\mathrm{r}}-\mathrm{u}_{\mathrm{r}}$ as elements in $\mathrm{R}[\mathrm{X}], \mathrm{R}=\mathrm{K}[\mathrm{u}]$, and calculate a Gröbner basis $\Gamma$ of the ideal $\left(P_{1}-u_{1}, \cdots, P_{r}-u_{r}\right)$ with the total-degree order for both $X_{1}, \ldots, X_{n}$ and $u_{1}, \ldots, u_{r}$;

Return: Return $\Gamma \cap \mathrm{K}[\mathrm{u}]$.

\subsection{Representing a polynomial by other polynomials}

Given polynomials $\mathrm{P}$ and $\mathrm{P}_{1}, \ldots, \mathrm{P}_{\mathrm{r}}$ in $\mathrm{K}[\mathrm{X}]$, we want to determine whether there exists a polynomial $Q\left(u_{1}, \ldots, u_{r}\right)$ in $K\left[u_{1}, \ldots, u_{r}\right]$ such that $P=Q\left(P_{1}, \ldots, P_{r}\right)$, and we want to determine $Q$ when exists. An algorithm performing this calculation will be quite useful for simplifying large polynomials. It is easy to see that this calculation is a special case of calculating algebraic relations described in 3.3 . Therefore, we have the following algorithm.

Algorithm D (polynomial composition).

Input : Polynomials $\mathrm{P}, \mathrm{P}_{1}, \ldots, \mathrm{P}_{\mathrm{r}}$ in $\mathrm{K}[\mathrm{X}]$;

Output: Polynomial $Q\left(u_{1}, \ldots, u_{r}\right)$, if exists, such that $P=Q\left(P_{1}, \ldots, P_{r}\right)$;

Method: Treat $P, P_{1}, \ldots, P_{r}$ as elements in $R[X], R=K[u]$, and calculate a Gröbner basis $\Gamma$ of $\left(P_{1}-u_{1}, \cdots, P_{r}-u_{r}\right)$ with the total-degree order for both $\mathrm{X}_{1}, \ldots, \mathrm{X}_{\mathrm{n}}$ and $\mathrm{u}_{1}, \ldots, \mathrm{u}_{\mathrm{r}}$. Then, $\mathrm{P} \underset{\mathrm{r}}{\longrightarrow} \mathrm{Q}$;

Return: If $Q \varepsilon \mathrm{K}[\mathrm{u}]$ then return $Q$ else return NIL.// 


\section{\$4. Devices for efficient elimination}

Although we have formulated the variable elimination as the construction of a Gröbner basis, we need not always calculate the full set of Gröbner basis but may stop the computation when the required elimination is accomplished. For example, in the calculation of U-resultant we may stop the computation when all the variables $X_{1}, \ldots, X_{n}$ are eliminated. (Then, the U-resultant calculated may contain an extra monomial factor.) This device will save the computation time drastically, as we will see from the actual timing data. Note that, with Choice 2 given in $\$ 3$, the required elimination will be performed by avoiding wasteful computation as far as possible.

The second device is the removal of monomial factors from the S-polynomials constructed, which is quite easy to execute. For example, monomial factors in algebraic relations are meaningless and we can remove them. Note that, even if such a monomial factor is meaningful, we can often remove it so long as the removed factor is processed suitably. For example, suppose $P_{i}=\widetilde{P}_{i} X_{k}^{a}$ in Algorithm $A$, then we can split the system of algebraic equations into two systems as $\left\{P_{1}=0, \ldots\right.$ $\left.P_{i-1}=0, \tilde{P}_{i}=0, P_{i+1}=0, \ldots, P_{r}=0\right\}$ and $\left\{P_{1}=0, \ldots, P_{i-1}=0, X_{k}^{a}=0, P_{i+1}=0, \ldots, P_{r}=0\right\}$ Hence, only if the latter system is also solved, we can remove the monomial factor $X_{k}^{a}$ from $P_{i}$.

In the current implementation of our Gröbner basis package, the user can choose one of the following three modes for controlling the truncation of computation.

(TO) No truncation (default mode);

(T1) Truncate the computation when variables $\mathrm{X}_{1}, \ldots \mathrm{X}_{\mathrm{n}}$ are eliminated;

(T2) Truncate the computation when variables $X_{1}, \ldots, X_{n}$ and parameters $u_{1}, \ldots$ $u_{m-1}$ are eliminated (the final polynomial is in $K\left[u_{m}\right]$ ).

Furthermore, the user can choose one of the following three modes for controlling the removal of the monomial factors.

(RO) No removal (default mode);

(R1) Monomial factors in $\mathrm{K}[\mathrm{u}]$ are removed;

(R2) Monomial factors in $\mathrm{K}[\mathrm{u}, \mathrm{X}]$ are removed. 
Including the above-mentioned devices, the algorithms given in the previous section can be improved as follows.

\section{Algorithm $A^{\prime}$ (reducing algebraic equations).}

Perform the second step of Algorithm A with modes (T1) and (R2), and calculate $Q_{1}\left(X_{1}\right)$ by eliminating, for example, $X_{2}$ from elements in the set $\Gamma_{2}$. (The removed factors should be saved for the later computation.)

\section{Algorithm $B^{\prime}$ (U-resultant).}

Perform Algorithm B with modes (T1) and (R1).

Algorithm C' (algebraic relations).

Perform Algorithm $\mathrm{C}$ with modes (T1) and (R1).

Let us show the effectiveness of the above-mentioned devices by several examples. The test has been done by using a Gröbner basis package on the Japanese algebra system GAL. The test problems are as follows.

Problem 1 (reducing a system of algebraic equations).

$$
\begin{aligned}
& F_{1}=2\left(X_{4}^{2}+X_{3}^{2}+X_{2}^{2}+X_{1}^{2}\right)-X_{1}=0, \\
& F_{2}=2\left(X_{4} X_{3}+X_{3} X_{2}+X_{2} X_{1}\right)-X_{2}=0 \\
& F_{3}=2\left(X_{4} X_{2}+X_{3} X_{1}\right)+X_{2}^{2}-X_{3}=0, \\
& F_{4}=2\left(X_{4}+X_{3}+X_{2}\right)+X_{1}-1=0 .
\end{aligned}
$$

This problem is taken from a theory of spin grass by Katsura et al.

Problem 2 (calculating a U-resultant).

$$
\mathrm{F}_{1}=\mathrm{X}_{1}^{2}+\mathrm{X}_{2}^{2}-2=0, \quad \mathrm{~F}_{2}=\mathrm{X}_{1} \mathrm{X}_{2}-1=0 \text {. }
$$

Adding $\mathrm{F}_{0}=\mathrm{u}_{0}+\mathrm{u}_{1} \mathrm{X}_{1}+\mathrm{u}_{2} \mathrm{X}_{2}=0$ to the above system, we can calculate the following polynomial as the U-resultant:

$$
\begin{aligned}
U\left(u_{0}, u_{1}, u_{2}\right) & =u_{0}^{4}-2 u_{0}^{2} u_{1}^{2}-4 u_{0}^{2} u_{1} u_{2}-2 u_{0}^{2} u_{2}^{2} \\
& +u_{1}^{4}+4 u_{1}^{3} u_{2}+6 u_{1}^{2} u_{2}^{2}+4 u_{1} u_{2}^{3}+u_{2}^{4} .
\end{aligned}
$$

Problem 3 (calculating an algebraic relation).

$$
\begin{aligned}
& \mathrm{P}_{1}=\left(\mathrm{X}_{1}^{6}+\mathrm{X}_{2}^{6}\right)+522\left(\mathrm{X}_{1}^{5} \mathrm{X}_{2}-\mathrm{X}_{1} \mathrm{X}_{2}^{5}\right)-10005\left(\mathrm{X}_{1}^{4} \mathrm{X}_{2}^{2}+\mathrm{X}_{1}^{2} \mathrm{X}_{2}^{4}\right) \\
& \mathrm{P}_{2}=-\left(\mathrm{X}_{1}^{4}+\mathrm{X}_{2}^{4}\right)+228\left(\mathrm{X}_{1}^{3} \mathrm{X}_{2}-\mathrm{X}_{1} \mathrm{X}_{2}^{3}\right)-494 \mathrm{X}_{1}^{2} \mathrm{X}_{2}^{2}, \\
& \mathrm{P}_{3}=\mathrm{X}_{1} \mathrm{X}_{2}\left(\mathrm{X}_{1}^{2}+11 \mathrm{X}_{1} \mathrm{X}_{2}-\mathrm{X}_{2}^{2}\right)^{5} .
\end{aligned}
$$

The algebraic relation of these polynomials is $\mathrm{P}_{1}^{2}+\mathrm{P}_{2}^{3}-1728 \mathrm{P}_{3}=0$. This problem 
is taken from a Klein's book discussing the symmetry of regular polyhedra.

Problem 4 (polynomial composition).

$$
\begin{aligned}
& P_{1}=x_{1}^{2} x_{2}+2 x_{1}^{2}-3 x_{1} x_{2}+5 x_{2} \\
& P_{2}=2 x_{1}^{3}-4 x_{1}^{2} x_{2}-3 x_{1} x_{2}^{2}+x_{2}^{3} \\
& P=-2 x_{1}^{9} x_{2}^{3}+4 x_{1}^{8} x_{2}^{4}+3 x_{1}^{7} x_{2}^{5}+\cdots+4 x_{1}^{2}-6 x_{1} x_{2}+10 x_{2} .
\end{aligned}
$$

Given these polynomials ( $P$ is composed of 49 terms), we derive a polynomial $Q\left(u_{1}, u_{2}\right)$ such that $P=Q\left(P_{1}, P_{2}\right)$. The form of $Q$ in this example is

$$
Q\left(u_{1}, u_{2}\right)=-u_{1}^{3} u_{2}+u_{1} u_{2}^{2}+2 u_{1}-3 u_{2} \text {. }
$$

\begin{tabular}{|c|c|c|c|c|}
\hline Algorithm & Prob.1 & Prob.2 & Prob. 3 & Prob.4 \\
\hline A-D & 17.080 & 1.136 & 251 & 115 \\
\hline$A^{\prime}-C^{\prime}$ & 690 & 65 & 122 & $* * * *$ \\
\hline
\end{tabular}

Table I. Timing data (in milliseconds)

Table I shows the timing data, where the computation is done by GAL on a FACOM-M380 computer. We see that the truncation mode is often quite effective. This effectiveness is due partly to Choice 1 given in $\$ 3$ and partly to skipping the termination check in Buchberger's procedure. For Problem 2, for example, the elimination has been performed after constructing $11 \mathrm{~S}$-polynomials while we must construct $16 \mathrm{~S}$-polynomials for the Gröbner basis calculation. The removal mode is effective only for Problem 2 in our test. In mode (R1), we obtain the U-resultant just when $\mathrm{X}_{1}$ and $\mathrm{X}_{2}$ are eliminated, through successive removal of monomials $u_{2}, u_{1}$, and $u_{1}$. On the other hand, in mode $(R 0)$, we obtain $u_{1}^{2} u_{2} \cdot U\left(u_{0}, u_{1}, u_{2}\right)$ just when $X_{1}$ and $\mathrm{X}_{2}$ have been eliminated, and we have to construct 19 more $\mathrm{S}$-polynomials to get $\mathrm{U}\left(\mathrm{u}_{0}, \mathrm{u}_{1}, \mathrm{u}_{2}\right)$. We have also solved Problem 2 by applying Algorithm 2 with modes (TO) and (R1) (i.e., no truncation but removal of monomial factors), and the computation time was 707 milliseconds. This shows that the removal mode is also effective considerably.

Although the above test is restricted within a small number of examples which are of small-sized, we have seen that our devices are quite effective in many 
cases. The devices will become more effective for larger-sized problems.

Acknowledgement. The author thanks Prof. Kobayashi and Mr. Furukawa for stimulating discussions and comments.

\section{References}

[Ar\&Se84] D. Arnon and T. Sederberg, "Implicit equations for parametric surfaces by Gröbner basis", Proc. of 1984 MACSYMA User's Conf. (ed. V.E. Golden), General Electric, pp. 431-435.

[Buch65] B. Buchberger, "An algorithm for finding a basis for the residue class ring of a zero-dimensional polynomial ideal (German)", Ph.D. Thesis, Math. Inst., Univ. of Innsbruck (Austria), 1965.

[Buch84] B. Buchberger, "Gröbner bases : An algorithmic method in polynomial ideal theory", in Recent Trends in Multidimensional Systems Theory (ed. R. Bose), Reidel, 1984.

[Ka\&Ka84] A. Kandri-Rody and D. Kapur, "Algorithms for computing Gröbner bases of polynomial ideals over various Euclidean rings". Proc. of EUROSAM '84 (ed. J. Fitch), 1984 (Lecture Notes Comp. Sci. 174, Springer-Verlag), pp. 195-206.

[K\&F\&F86] H. Kobayashi, T. Fujise, and A. Furukawa, "Solving systems of algebraic equations by general elimination method", preprint of Dept. Math.. Nihon Univ.. Tokyo, 1986 (to be published).

[Lauer76] M. Lauer, "Canonical representatives for the residue classes of a polynomial ideal (German)", Diploma Thesis, Dept. Math., Univ. Kaiserslautern, 1976.

[Laza77] D. Lazard, "Algèbre linéaire sur $\mathrm{K}\left[\mathrm{x}_{1}, \mathrm{x}_{2}, \ldots, \mathrm{x}_{\mathrm{n}}\right]$ et éllimination". Bull. Soc. Math. France, No. 105 (1977), pp. 165-190.

[Mori86] S. Moritsugu, "On solving a system of algebraic equations by using Gröbner basis", preprint of Dept. Inf. Sci., Univ. of Tokyo, Tokyo, 1987. 